\title{
ESPÉCIES ARBÓREAS DE UM TRECHO DE FLORESTA ATLÂNTICA DO MUNICÍPIO DE ANTONINA, PARANÁ, BRASIL
}

\author{
Marilia Borgo $^{1}$, Gilberto Tiepolo ${ }^{2}$, Marcelo Reginato ${ }^{3}$, Yoshiko S. Kuniyoshi ${ }^{4}$, Franklin Galvão ${ }^{5}$, \\ Robson L. Capretz ${ }^{6}$, Victor P. Zwiener ${ }^{7}$ \\ ${ }^{1}$ Bióloga, Dra ., SPVS, Curitiba, PR, Brasil - maborgo@yahoo.com \\ ${ }^{2}$ Eng. Florestal, M.Sc., TNC do Brasil, Curitiba, PR, Brasil - gtiepolo@tnc.org \\ ${ }^{3}$ Biólogo, M.Sc., The New York Botanical Garden, New York, USA - reginatobio@yahoo.com.br \\ ${ }^{4}$ Naturalista, Dr ${ }^{\mathrm{a}}$., Depto. de Ciências Florestais, UFPR, Curitiba, PR, Brasil - yoshiko@ufpr.br \\ ${ }^{5}$ Eng. Florestal, Dr., Depto. de Ciências Florestais, UFPR, Curitiba, PR, Brasil - fgalvao@ufpr.br \\ ${ }^{6}$ Ecólogo, Dr., SPVS, Curitiba, PR, Brasil - robsoncapretz@ spvs.org.br \\ ${ }^{7}$ Biólogo, M.Sc., SPVS, Curitiba, PR, Brasil - victorpz_bio@yahoo.com.br
}

Recebido para publicação: 20/12/2010 - Aceito para publicação: 27/07/2011

\begin{abstract}
Resumo
Com o objetivo de subsidiar futuros estudos de dinâmica da vegetação e efeitos das mudanças climáticas, realizou-se um levantamento florístico do componente arbóreo em área de Floresta Ombrófila Densa da Reserva Natural do Rio Cachoeira, em Antonina, Paraná. O levantamento foi realizado em 187 parcelas permanentes, que representam 23,5 ha amostrados. Foram identificadas 306 espécies distribuídas em 68 famílias. A zoocoria foi a síndrome de dispersão mais comum $(81,7 \%$ das espécies). Espécies tolerantes à sombra superaram discretamente as não tolerantes $(50,3 \% \mathrm{e}$ $49,7 \%$, respectivamente). Espécies de distribuição geográfica ampla representaram quase metade do total $(48,4 \%)$. Desse conjunto florístico, 21 espécies encontram-se sob alguma categoria de risco de extinção, o que representa $6,9 \%$ do universo encontrado, valor maior que o verificado na maioria dos estudos conduzidos nessa mesma formação (em torno de 2\%). Essa situação reflete os efeitos da pressão contínua a que a floresta está sujeita, onde a superexplotação de essências nativas ao longo dos anos, associada à deterioração da floresta por meio da fragmentação e da perda de hábitats, resulta em maior risco à conservação de espécies do bioma.
\end{abstract}

Palavras-chave: Floresta Ombrófila Densa; florística; espécies ameaçadas; síndromes de dispersão.

\begin{abstract}
Tree species from an Atlantic Forest remnant in Antonina, Paraná, Southern Brazil. Aiming to support new vegetation dynamics and climate change studies, a floristic survey of the tree species was conducted in the Atlantic Forest at Rio Cachoeira Natural Reserve, in Antonina municipality, Southern Brazil. The survey was intensified in 187 permanent plots, representing 23.5 ha of sampled area. The floristic collection was represented by 306 tree species, distributed in 68 families. Zoochory was the most common dispersion syndrome (81.7\%). According to the shadow tolerance, $50.3 \%$ of the species were tolerant, while non-tolerant species were $49.7 \%$. Wide geographical distribution species represented almost half of all this floristic set $(48.4 \%)$. Threatened species were $6.9 \%$ of all species found (21), a higher value than that one reported to other studies on this forest type (around $2 \%$ ). This situation shows the effects of continuous pressure on the forest, as the over exploitation of the vegetation through the years, associated to the forest damage by fragmentation and habitat loss process leads to the higher risks for species conservation on this biome.

Keywords: Ombrophilous Dense Forest; floristic composition; threatened species; dispersion ways.
\end{abstract}

\section{INTRODUÇÃO}

A Floresta Atlântica sensu stricto, conhecida como Floresta Ombrófila Densa (INSTITUTO BRASILEIRO DE GEOGRAFIA E ESTATÍSTICA/IBGE, 1992), é uma das tipologias de maior diversidade mundial. Considerada como um dos centros de biodiversidade ameaçados do planeta (hotspots), ela detém cerca de 20.000 espécies (MYERS et al., 2000). Não fosse só a grande riqueza de 
espécies - Leite e Klein (1990) registraram a ocorrência de 700 espécies arbóreas em Santa Catarina - a formação também apresenta um elevado grau de endemismos (GIULIETTI et al., 2005; FIASCHI; PIRANI, 2009; CENTROS DE ENDEMISMO DE PLANTAS VASCULARES NA MATA ATLÂNTICA/CEPVMA, 2010).

Esse tipo vegetacional, exuberante nas formas de vida e nas fisionomias, é resultado da combinação de um grande número de condições (altitude, umidade, precipitação, características físicas dos solos, disponibilidade de água, radiação e nutrientes) (RODERJAN et al., 2002; PRÓ-ATLÂNTICA, 2005). Distribuía-se originalmente por quase toda a costa brasileira (FUNDAÇÃO SOS MATA ATLÂNTICA; INPE, 2008) e os trechos melhor preservados nas regiões Sul e Sudeste que restaram são produto da restrição natural do avanço da ocupação humana sobre a floresta. As faces bastante íngremes da Serra do Mar impediram que houvesse uma expansão maior dos centros urbanos, situados, em sua maioria, na região costeira (PINTO; BRITO, 2003). Ainda assim, hoje restam pouco mais de $12 \%$ de florestas relativamente bem conservadas, e um dos poucos trechos contínuos de maior expressão estão entre o Paraná e São Paulo (RIBEIRO et al., 2009).

Avaliações abordando riqueza da formação trazem informações bastante variadas, mas sempre reforçando a extrema riqueza desse tipo vegetacional. No Paraná, Stellfeld (1949) e Maack (1968) foram pioneiros ao descrever aspectos gerais de florística e fisionomia dessa formação. Após eles, estudos foram conduzidos em várias feições da floresta, abordando grupos específicos da flora (SCHORN, 1992; SILVA, 1994; RODERJAN, 1994; GUAPYASSÚ, 1994; DITTRICH et al., 2005; KOZERA et al., 2009).

Diante da perspectiva de pesquisa em longo prazo estabelecida com a implantação de um projeto de avaliação da dinâmica de biomassa da Floresta Atlântica na região de Antonina, o presente trabalho buscou analisar o conjunto de espécies que compõem a flora arbórea da Reserva Natural do Rio Cachoeira e averiguar se há distinção no conjunto de espécies quanto à dispersão, tolerância à sombra e distribuição geográfica que elas apresentam. Essas informações são cruciais ao desenvolvimento de novas pesquisas na área, em especial as que envolvem restauração florestal e relações ecológicas das comunidades vegetais, tão importantes para ações de conservação do bioma.

\section{MATERIAL E MÉTODO}

O levantamento florístico foi realizado na Reserva Natural Rio Cachoeira (RNRC), área protegida com 8.600 ha, de propriedade da Sociedade de Pesquisa de Vida Selvagem e Educação Ambiental (SPVS). A RNRC está localizada no município de Antonina, litoral norte do Paraná (2519'15' S e 48 42'24' W), dentro da Área de Proteção Ambiental de Guaraqueçaba.

O clima da região, segundo sistema de Köppen, é o Cfa, subtropical úmido mesotérmico, com temperatura média de $20,6^{\circ} \mathrm{C}$, precipitação média anual de $2.517 \mathrm{~mm}$ e umidade relativa média de $85 \%$ (INSTITUTO PARANAENSE DE DESENVOLVIMENTO ECONÔMICO E SOCIAL/IPARDES, 2001). O relevo local é diverso, variando de plano a forte ondulado (ou escarpado), ocorrendo predominantemente Cambissolos, Argissolos, Neossolos Flúvicos e Neossolos Litólicos (SPVS; TNC, 2002). Seguindo classificação do IBGE (1992), a RNRC é recoberta por diferentes estágios sucessionais da Floresta Ombrófila Densa nas formações Aluvial, de Terras Baixas e Submontana, além de Formações Pioneiras de Influência Fluvial e Fluviomarinha, em uma faixa altitudinal que varia de 0 a $540 \mathrm{~m}$ s.n.m.

O estudo florístico ocorreu entre os anos de 2002 e 2009 e baseou-se em coletas realizadas principalmente em 187 parcelas circulares permanentes (raio $=20 \mathrm{~m}$; DAP $\geq 5 \mathrm{~cm}$ ), utilizadas em inventário de estoque de carbono, que foram distribuídas paralelamente às trilhas que cortam a reserva. As parcelas foram alocadas de forma a contemplar todos os estágios sucessionais das florestas de planície e encosta (formações Submontana, Terras Baixas e Aluvial), oriundos de diferentes formas e graus de interferência (em especial o corte raso e uso posterior para agricultura ou pastagens destinadas à bubalinocultura, ou então extração seletiva de essências nativas). Essas parcelas representaram uma área amostral de 23,5 ha, sendo também coletado material fértil de indivíduos encontrados em incursões ao longo dos $150 \mathrm{~km}$ de trilhas da reserva.

As determinações foram feitas através de consulta aos herbários do Museu Botânico Municipal de Curitiba (MBM), do Departamento de Botânica (UPCB) e da Escola de Florestas (EFC) da Universidade Federal do Paraná, literatura específica e consulta a especialistas. O material fértil foi 
enviado para registro aos herbários MBM e UPCB (números de registro podem ser consultados no SpeciesLink - http://splink.cria.org.br). O sistema de classificação utilizado foi o proposto por Angiosperm Phylogeny Group (APG) III (2009). A distribuição geográfica (Ampla: em diferentes formações vegetais brasileiras; FA: ao longo da área de ocorrência da Floresta Atlântica; FA-S: restrita à Floresta Atlântica das regiões Sul e Sudeste do Brasil) seguiu o Jardim Botânico do Rio de Janeiro/JBRJ (2010). A validade dos binômios científicos foi verificada junto ao JBRJ (2010), e o status de conservação junto a IUCN (2011) e Brasil (2008).

De acordo com observações feitas em campo, para cada espécie foram indicadas a categoria ecológica sucessional (não tolerantes à sombra - incapazes de se desenvolver em trechos sujeitos a sombreamento contínuo; e tolerantes à sombra - que completam seu ciclo vital em ambientes sem incidência direta de luz) (HARTSHORN, 1978) e a síndrome de dispersão (anemocóricas: diásporos alados, plumosos ou em forma de balão ou poeira; zoocóricas: diásporos com atrativos e/ou fontes alimentares, com estruturas adesivas como ganchos, cerdas, espinhos; autocóricas: espécies barocóricas ou com dispersão explosiva), seguindo Van der Pijl (1982).

\section{RESULTADOS E DISCUSSÃO}

No componente arbóreo das diferentes fases serais da Floresta Ombrófila Densa na RNRC, foram encontradas 306 espécies distribuídas em 68 famílias (Tabela 1). Myrtaceae (60 espécies), Fabaceae (32), Lauraceae (23), Melastomataceae (16), Rubiaceae (11) e Moraceae (10) englobaram 49,7\% do total de taxa encontrados. Dentro desse universo de espécies, 21 encontram-se citadas na IUCN Red List (IUCN, 2011), estando sob algum grau de ameaça de extinção. Das três espécies que constam na lista brasileira - Ocotea catharinensis, Ocotea odorifera e Euterpe edulis (BRASIL, 2008) -, apenas esta última, uma palmeira, não faz parte da lista da IUCN.

Praticamente não houve diferença no número de espécies tolerantes e não tolerantes à sombra (50,3\% e $49,7 \%$, respectivamente). A zoocoria foi a síndrome de dispersão mais comum, ocorrendo em $81,7 \%$ das espécies registradas, seguida pela anemocoria $(11,4 \%)$ e pela autocoria $(6,9 \%)$.

Tabela 1. Espécies da sinúsia arbórea em Floresta Ombrófila Densa da Reserva Natural Rio Cachoeira, Antonina, Paraná, Brasil, com respectivos nomes vulgares, síndrome de dispersão (ZOOzoocoria, ANE-anemocoria, AUT-autocoria), categoria ecológica - CE (NT-não tolerante à sombra; T-tolerante à sombra) e distribuição geográfica (Amp: ampla; FA: Floresta Atlântica; FA-S: Floresta Atlântica das regiões Sul e Sudeste). *Indica espécie ameaçada.

Table 1. Tree species from Atlantic Forest of Rio Cachoeira Natural Reserve, Paraná, Southern Brazil, with local names, dispersion syndrome (ZOO-zoochory; ANE-wind dispersion; AUTautochory), ecological category (NT-pioneer; T-shadow tolerant), geographic distribution (Amp: wide distribution; FA: Atlantic Forest; FA-S: Southeast and Southern regions of Atlantic Forest). *Indicates threatened species.

\begin{tabular}{llcccc}
\hline Família & Espécie & Nome comum & Disper. & CE & Dist. \\
\hline Anacardiaceae & Schinus terebinthifolius Raddi & aroeira & ZOO & NT & Amp \\
Anacardiaceae & Tapirira guianensis Aubl. & cupiúva & ZOO & NT & Amp \\
Annonaceae & Annona glabra L. & araticum-do-brejo & ZOO & NT & Amp \\
Annonaceae & Annona neosericea H. Rainer & ariticum & ZOO & NT & FA-S \\
Annonaceae & Annona sylvatica A. St.-Hil. & ariticum & ZOO & T & Amp \\
Annonaceae & Guatteria australis A. St.-Hil. & ariticum & ZOO & T & FA \\
Annonaceae & Xylopia brasiliensis Spreng. & pendoveira & ZOO & T & FA-S \\
Apocynaceae & Aspidosperma pyricollum Müll. Arg. & perovana & ANE & T & Amp \\
Apocynaceae & Aspidosperma ramiflorum Müll. Arg. & peroba-vermelha & ANE & T & FA \\
Apocynaceae & Malouetia cestroides (Nees ex Mart.) Müll. Arg. & goerana & ANE & NT & Amp \\
Apocynaceae & Tabernaemontana catharinensis A. DC. & leiteiro & ZOO & NT & Amp \\
Aquifoliaceae & Ilex dumosa Reissek & caúna & ZOO & NT & Amp \\
Aquifoliaceae & Ilex integerrima Reissek & caúna & ZOO & NT & FA \\
Aquifoliaceae & Ilex pseudobuxus Reissek & caúna & ZOO & NT & Amp \\
Aquifoliaceae & Ilex sp. & caúna & ZOO & NT & - \\
\hline
\end{tabular}




\begin{tabular}{|c|c|c|c|c|c|}
\hline Araliaceae & Schefflera angustissima (Marchal) Frodin & mandiocão & $\mathrm{ZOO}$ & NT & FA-S \\
\hline Araliaceae & $\begin{array}{l}\text { Schefflera morototoni (Aubl.) Maguire, Steyerm. } \\
\& \text { Frodin }\end{array}$ & mandiocão & $\mathrm{ZOO}$ & NT & Amp \\
\hline Arecaceae & Astrocaryum aculeatissimum (Schott) Burret* & brejaúba & $\mathrm{ZOO}$ & $\mathrm{T}$ & FA \\
\hline Arecaceae & Attalea dubia (Mart.) Burret & indaiá & $\mathrm{ZOO}$ & $\mathrm{T}$ & FA-S \\
\hline Arecaceae & Bactris setosa Mart. & tucum & $\mathrm{ZOO}$ & $\mathrm{T}$ & Amp \\
\hline Arecaceae & Bactris sp. & tucum-mirim & $\mathrm{ZOO}$ & $\mathrm{T}$ & - \\
\hline Arecaceae & Euterpe edulis Mart. & palmito-jussara & $\mathrm{ZOO}$ & $\mathrm{T}$ & Amp \\
\hline Arecaceae & Geonoma elegans Mart. & guaricana & $\mathrm{ZOO}$ & $\mathrm{T}$ & FA \\
\hline Arecaceae & Geonoma gamiova Barb. Rodr. & guaricana & $\mathrm{ZOO}$ & $\mathrm{T}$ & FA-S \\
\hline Arecaceae & Geonoma schottiana Mart. & palha & $\mathrm{ZOO}$ & $\mathrm{T}$ & FA-S \\
\hline Arecaceae & Syagrus rommanzoffiana Cham. & jerivá & $\mathrm{ZOO}$ & NT & Amp \\
\hline Asteraceae & Baccharis sp. & vassourinha & ANE & NT & - \\
\hline Asteraceae & Piptocarpha cf. axillaris (Less.) Baker & cambará-branco & ANE & NT & Amp \\
\hline Asteraceae & Piptocarpha sp. & cambará & ANE & NT & - \\
\hline Asteraceae & Vernonanthura sp. & vassoura & ANE & NT & - \\
\hline Bignoniaceae & Cybistax antisyphilitica (Mart.) Mart. & ipê-verde & ANE & NT & Amp \\
\hline Bignoniaceae & Handroanthus umbellatus (Sond.) Mattos & ipê-do-brejo & ANE & NT & Amp \\
\hline Bignoniaceae & Handroanthus sp. & ipê-amarelo & ANE & $\mathrm{T}$ & - \\
\hline Bignoniaceae & Jacaranda puberula Cham. & carova & ANE & NT & FA-S \\
\hline Bignoniaceae & Tabebuia cassinoides DC. & caxeta & ANE & NT & FA-S \\
\hline Boraginaceae & Cordia cf. ecalyculata Vell. & jurutê & $\mathrm{ZOO}$ & NT & FA \\
\hline Boraginaceae & Cordia silvestris Fresen. & jurutê & $\mathrm{ZOO}$ & NT & FA-S \\
\hline Burseraceae & Protium kleinii Cuatr. & almesca & $\mathrm{ZOO}$ & $\mathrm{T}$ & FA-S \\
\hline Canellaceae & Cinnamodendron dinisii Schwacke & pimenteira & $\mathrm{ZOO}$ & $\mathrm{T}$ & FA-S \\
\hline Cannabaceae & Trema micrantha (L.) Blume & crindiúva & $\mathrm{ZOO}$ & NT & Amp \\
\hline Cardiopteridaceae & Citronella paniculata (Mart.) Howard & & $\mathrm{ZOO}$ & NT & Amp \\
\hline Caricaceae & Jacaratia spinosa (Aubl.) A. DC. & mamão-bravo & $\mathrm{ZOO}$ & NT & Amp \\
\hline Celastraceae & Maytenus gonoclada Mart. & & $\mathrm{ZOO}$ & $\mathrm{T}$ & Amp \\
\hline Celastraceae & Maytenus robusta Reissek & & $\mathrm{ZOO}$ & $\mathrm{T}$ & Amp \\
\hline Celastraceae & Maytenus schumaniana Loes. & coração-de-negro & $\mathrm{ZOO}$ & $\mathrm{T}$ & FA \\
\hline Chloranthaceae & Hedyosmum brasiliense Mart. & cidreira & $\mathrm{ZOO}$ & NT & Amp \\
\hline Chrysobalanaceae & Hirtella hebeclada Moric. ex A.P. DC. & & $\mathrm{ZOO}$ & $\mathrm{T}$ & Amp \\
\hline Clethraceae & Clethra scabra Pers. & carne-de-vaca & AUT & NT & Amp \\
\hline Clusiaceae & Calophyllum brasiliense Cambess. & guanandi & $\mathrm{ZOO}$ & $\mathrm{T}$ & Amp \\
\hline Clusiaceae & Clusia criuva Cambess. & mangue-do-mato & $\mathrm{ZOO}$ & NT & FA \\
\hline Clusiaceae & Garcinia gardneriana (Planch. \& Triana) Zappi & bacopari & $\mathrm{ZOO}$ & $\mathrm{T}$ & Amp \\
\hline Combretaceae & Buchenavia kleinii Exell* & guarajuva & $\mathrm{ZOO}$ & NT & FA-S \\
\hline Cunnoniaceae & Weinmannia paulliniifolia Pohl ex Ser. & gramimunha & ANE & NT & FA-S \\
\hline Ebenaceae & Diospyros sp. & & $\mathrm{ZOO}$ & NT & - \\
\hline Elaeocarpaceae & Sloanea garckeana K. Schum. & laranjeira-do-mato & AUT & $\mathrm{T}$ & Amp \\
\hline Elaeocarpaceae & Sloanea guianensis (Aubl.) Benth. & laranjeira-imbiúva & AUT & $\mathrm{T}$ & Amp \\
\hline Erythroxylaceae & Erythroxylum amplifolium (Mart.) Schult. & cocão & $\mathrm{ZOO}$ & NT & Amp \\
\hline Erythroxylaceae & Erythroxylum cuspidifolium Mart. & cocão & $\mathrm{ZOO}$ & NT & FA \\
\hline Erythroxylaceae & Erythroxylum deciduum A. St.-Hil. & cocão & $\mathrm{ZOO}$ & NT & Amp \\
\hline Euphorbiaceae & Actinostemon concolor Müll. Arg. & tabocuvão & AUT & NT & Amp \\
\hline Euphorbiaceae & Alchornea glandulosa Poepp. & tapiá, tapiaeiro & $\mathrm{ZOO}$ & NT & Amp \\
\hline Euphorbiaceae & Alchornea triplinervia (Spreng.) Müll.Arg. & tapiá, tapiaeiro & $\mathrm{ZOO}$ & NT & Amp \\
\hline Euphorbiaceae & Aparisthmium cordatum (A. Juss.) Baill. & & $\mathrm{ZOO}$ & NT & Amp \\
\hline Euphorbiaceae & Maprounea brasiliensis A. St.-Hil. & cauvitinga & $\mathrm{ZOO}$ & NT & Amp \\
\hline Euphorbiaceae & Pachystroma longifolium I.M. Johnst. & guarapicica & AUT & $\mathrm{T}$ & Amp \\
\hline Euphorbiaceae & Pausandra morisiana (Casar.) Radlk. & & AUT & $\mathrm{T}$ & FA \\
\hline Euphorbiaceae & Sapium glandulosum (L.) Morong & leiteiro & $\mathrm{ZOO}$ & NT & Amp \\
\hline Euphorbiaceae & Tetrorchidium rubrivenium Poepp. \& Endl. & & $\mathrm{ZOO}$ & NT & Amp \\
\hline Fabaceae & Andira anthelmia (Vell.) Benth. & jacarandá-lombriga & $\mathrm{ZOO}$ & $\mathrm{T}$ & Amp \\
\hline
\end{tabular}




\begin{tabular}{|c|c|c|c|c|c|}
\hline Fabaceae & Bauhinia forficata Link & pata-de-vaca & AUT & NT & FA \\
\hline Fabaceae & $\begin{array}{l}\text { Centrolobium microchaete (Mart. ex Benth.) H.C. } \\
\text { Lima }\end{array}$ & araribá & ANE & NT & FA \\
\hline Fabaceae & Copaifera trapezifolia Hayne & óleo & $\mathrm{ZOO}$ & $\mathrm{T}$ & FA \\
\hline Fabaceae & Dahlstedtia pentaphylla (Taub.) Burkart & bico-de-papagaio & AUT & $\mathrm{T}$ & FA-S \\
\hline Fabaceae & Dalbergia brasiliensis Vogel & & ANE & NT & Amp \\
\hline Fabaceae & Dalbergia frutescens (Vell.) Britton & rabo-de-macaco & ANE & $\mathrm{T}$ & Amp \\
\hline Fabaceae & Erytrina speciosa Andrews & mulungu & AUT & NT & Amp \\
\hline Fabaceae & Inga edulis Mart. & ingá & $\mathrm{ZOO}$ & NT & Amp \\
\hline Fabaceae & Inga marginata Willd. & ingá-feijão & $\mathrm{ZOO}$ & NT & Amp \\
\hline Fabaceae & Inga sessilis DC. & ingá-ferradura & $\mathrm{ZOO}$ & NT & Amp \\
\hline Fabaceae & Inga sp. & ingá & $\mathrm{ZOO}$ & NT & - \\
\hline Fabaceae & Inga striata Benth. & ingá & $\mathrm{ZOO}$ & NT & FA \\
\hline Fabaceae & Machaerium brasiliense Vogel & amendoinzeiro & ANE & NT & Amp \\
\hline Fabaceae & Machaerium hatschbachii Rudd & & ANE & NT & FA-S \\
\hline Fabaceae & Machaerium hirtum (Vell.) Stellfeld & & ANE & NT & Amp \\
\hline Fabaceae & Machaerium uncinatum (Vell.) Benth. & & ANE & NT & FA \\
\hline Fabaceae & Mimosa bimucronata (DC.) Kuntze & maricá & ANE & NT & Amp \\
\hline Fabaceae & Myrocarpus frondosus Allemão* & caburê & ANE & $\mathrm{T}$ & FA \\
\hline Fabaceae & Ormosia arborea (Vell.) Harms & coronha & $\mathrm{ZOO}$ & $\mathrm{T}$ & Amp \\
\hline Fabaceae & Piptadenia gonoacantha (Mart.) Brenam & cauvitinga & ANE & NT & Amp \\
\hline Fabaceae & Platymiscium floribundum Vogel & jacarandá-rosa & ANE & NT & Amp \\
\hline Fabaceae & Pseudopiptadenia warmingii Benth. & cauvi & ANE & NT & FA \\
\hline Fabaceae & Pterocarpus rohrii Vahl & sangueiro & ANE & $\mathrm{T}$ & Amp \\
\hline Fabaceae & Schizolobium parahyba (Vell.) Blake & guapuruvu & AUT & NT & Amp \\
\hline Fabaceae & Senna cf. spectabilis (DC.) H.S.Irwin \& Barneby & & AUT & NT & Amp \\
\hline Fabaceae & Senna multijuga (L.C. Richard) H.S. Irwin \& Barneby & aleluia & AUT & NT & Amp \\
\hline Fabaceae & Senna oblongifolia (Vogel) H.S.Irwin \& Barneby & & AUT & NT & Amp \\
\hline Fabaceae & Senna pendula (Willd.) H.S.Irwin \& Barneby & & AUT & NT & Amp \\
\hline Fabaceae & Senna silvestris (Vell.) H.S.Irwin \& Barneby & araribá-da-capoeira & AUT & NT & Amp \\
\hline Fabaceae & Swartzia acutifolia Vogel & & $\mathrm{ZOO}$ & NT & Amp \\
\hline Fabaceae & Zolernia ilicifolia (Brongn.) Vogel & milho-seco & $\mathrm{ZOO}$ & $\mathrm{T}$ & Amp \\
\hline Humiriaceae & Humiriastrum sp. & & $\mathrm{ZOO}$ & $\mathrm{T}$ & - \\
\hline Humiriaceae & Vantanea compacta (Schinzl.) Cuatrec. & parapari & $\mathrm{ZOO}$ & $\mathrm{T}$ & Amp \\
\hline Lacistemataceae & Lacistema lucidum Schnizl. & & $\mathrm{ZOO}$ & $\mathrm{T}$ & FA-S \\
\hline Lamiaceae & Aegiphila integrifolia (Jacq.) Moldenke & tamanqueira & $\mathrm{ZOO}$ & NT & Amp \\
\hline Lamiaceae & Vitex polygama Cham. & tarumã & $\mathrm{ZOO}$ & $\mathrm{T}$ & Amp \\
\hline Lauraceae & Aiouea saligna Meisn. & canela & $\mathrm{ZOO}$ & $\mathrm{T}$ & Amp \\
\hline Lauraceae & Aniba firmula (Nees) $\mathrm{Mez}$ & & $\mathrm{ZOO}$ & $\mathrm{T}$ & FA \\
\hline Lauraceae & Cryptocarya mandioccana Meissn. & nhutinga & $\mathrm{ZOO}$ & $\mathrm{T}$ & FA \\
\hline Lauraceae & Cryptocarya aschersoniana $\mathrm{Mez}$ & nhutinga & $\mathrm{ZOO}$ & $\mathrm{T}$ & FA-S \\
\hline Lauraceae & Endlicheria paniculata (Spreng.) Macbr. & canela-frade & $\mathrm{ZOO}$ & $\mathrm{T}$ & Amp \\
\hline Lauraceae & Nectandra leucantha Nees & canela-amarela & $\mathrm{ZOO}$ & NT & FA-S \\
\hline Lauraceae & Nectandra megapotamica (Spreng.) Mez & canela-bosta & $\mathrm{ZOO}$ & NT & Amp \\
\hline Lauraceae & Nectandra membranacea Griseb. & canela-amarela & $\mathrm{ZOO}$ & NT & Amp \\
\hline Lauraceae & Nectandra oppositifolia Nees & canela-jussara & $\mathrm{ZOO}$ & NT & Amp \\
\hline Lauraceae & Nectandra puberula (Schott) Nees & canelinha & $\mathrm{ZOO}$ & NT & Amp \\
\hline Lauraceae & Nectandra reticulata (Ruiz \& Pav.) Mez & abacateiro-bravo & $\mathrm{ZOO}$ & NT & Amp \\
\hline Lauraceae & Ocotea aciphylla (Nees) Mez* & canela & $\mathrm{ZOO}$ & $\mathrm{T}$ & Amp \\
\hline Lauraceae & Ocotea catharinensis $\mathrm{Mez}^{*}$ & canela-preta & $\mathrm{ZOO}$ & $\mathrm{T}$ & FA-S \\
\hline Lauraceae & Ocotea dispersa (Nees \& Mart.) Mez & canela & $\mathrm{ZOO}$ & $\mathrm{T}$ & FA-S \\
\hline Lauraceae & Ocotea glaziovii $\mathrm{Mez}$ & canela & $\mathrm{ZOO}$ & $\mathrm{T}$ & - \\
\hline Lauraceae & Ocotea nunesiana (Vattimo-Gil) Baitello & canela & $\mathrm{ZOO}$ & $\mathrm{T}$ & FA-S \\
\hline Lauraceae & Ocotea odorifera (Vell.) Howher* & sassafrás & $\mathrm{ZOO}$ & $\mathrm{T}$ & Amp \\
\hline Lauraceae & Ocotea puberula Nees* & guaicá & $\mathrm{ZOO}$ & NT & Amp \\
\hline
\end{tabular}




\begin{tabular}{|c|c|c|c|c|c|}
\hline Lauraceae & Ocotea pulchella Mart. & canela-lageana & $\mathrm{ZOO}$ & NT & FA-S \\
\hline Lauraceae & Ocotea sp. & canela & $\mathrm{ZOO}$ & NT & - \\
\hline Lauraceae & Ocotea teleiandra (Meisn.) Mez & canela-pitanga & $\mathrm{ZOO}$ & $\mathrm{T}$ & FA-S \\
\hline Lauraceae & Ocotea tristis Mart. ex Nees & canela & $\mathrm{ZOO}$ & NT & FA-S \\
\hline Lauraceae & Persea sp. & pau-andrade & $\mathrm{ZOO}$ & NT & - \\
\hline Lecythidaceae & Cariniana estrellensis (Raddi) Kuntze & estopeira, jequitibá & ANE & $\mathrm{T}$ & Amp \\
\hline Magnoliaceae & Magnolia ovata P.Parm. & baguaçu & $\mathrm{ZOO}$ & $\mathrm{T}$ & Amp \\
\hline Malpighiaceae & Bunchosia pallescens Skottsb. & & $\mathrm{ZOO}$ & NT & FA-S \\
\hline Malpighiaceae & Byrsonima ligustrifolia A. St.-Hil. & murici & $\mathrm{ZOO}$ & NT & FA-S \\
\hline Malvaceae & Pseudobombax grandiflorum (Cav.) A.Robyns & embiruçu & ANE & NT & Amp \\
\hline Melastomataceae & Leandra dasytricha (A.Gray) Cogn. & & $\mathrm{ZOO}$ & NT & FA-S \\
\hline Melastomataceae & Leandra fragilis Cogn. & & $\mathrm{ZOO}$ & NT & FA \\
\hline Melastomataceae & Miconia cabucu Hoehne & pixiricão & $\mathrm{ZOO}$ & NT & FA-S \\
\hline Melastomataceae & Miconia carthacea Triana & pixiricão & $\mathrm{ZOO}$ & NT & FA \\
\hline Melastomataceae & Miconia cinerascens var. robusta Wurdack & pixiricão & $\mathrm{ZOO}$ & NT & FA-S \\
\hline Melastomataceae & Miconia cinnamomifolia (DC.) Naudin & pixiricão & $\mathrm{ZOO}$ & NT & FA \\
\hline Melastomataceae & Miconia cubatanensis Hoehne & pixirica & $\mathrm{ZOO}$ & NT & Amp \\
\hline Melastomataceae & Miconia dodecandra Cogn. & pixiricão & $\mathrm{ZOO}$ & NT & Amp \\
\hline Melastomataceae & Miconia fasciculata Gardner & pixirica & $\mathrm{ZOO}$ & NT & FA \\
\hline Melastomataceae & Miconia jucunda (DC.) Triana & pixirica & $\mathrm{ZOO}$ & NT & Amp \\
\hline Melastomataceae & Miconia latecrenata (DC.) Naudin & pixirica & $\mathrm{ZOO}$ & NT & FA \\
\hline Melastomataceae & Miconia pusiliflora (DC.) Naudin & pixirica & $\mathrm{ZOO}$ & NT & FA \\
\hline Melastomataceae & Miconia tristis ssp. australis Wurdack & & $\mathrm{ZOO}$ & NT & FA \\
\hline Melastomataceae & Mouriri chamissoana Cogn. & & $\mathrm{ZOO}$ & $\mathrm{T}$ & FA \\
\hline Melastomataceae & Tibouchina pulchra (Cham.) Cogn. & jacatirão & ANE & NT & FA-S \\
\hline Melastomataceae & Tibouchina trichopoda (DC.) Baill. & jacatirão-do-brejo & ANE & NT & FA-S \\
\hline Meliaceae & Cabralea canjerana (Vell.) Mart. & cajarana & $\mathrm{ZOO}$ & $\mathrm{T}$ & Amp \\
\hline Meliaceae & Cedrela fissilis Vell.* & cedro-rosa & ANE & NT & Amp \\
\hline Meliaceae & Guarea macrophylla Vahl & cafezeiro-bravo & $\mathrm{ZOO}$ & $\mathrm{T}$ & Amp \\
\hline Meliaceae & Trichilia lepidota Mart. & guacá & $\mathrm{ZOO}$ & $\mathrm{T}$ & FA \\
\hline Meliaceae & Trichilia pallens $\mathrm{Sw} *{ }^{*}$ & catiguá & $\mathrm{ZOO}$ & $\mathrm{T}$ & Amp \\
\hline Meliaceae & Trichilia silvatica DC.* & & $\mathrm{ZOO}$ & $\mathrm{T}$ & Amp \\
\hline Monimiaceae & Mollinedia argyrogyna Perkins* & & $\mathrm{ZOO}$ & $\mathrm{T}$ & Amp \\
\hline Monimiaceae & Mollinedia blumenaviana Perkins & & $\mathrm{ZOO}$ & $\mathrm{T}$ & FA-S \\
\hline Monimiaceae & Mollinedia schottiana Perkins & pau-andré & $\mathrm{ZOO}$ & $\mathrm{T}$ & FA \\
\hline Monimiaceae & Mollinedia uleana Perkins & pau-andré & $\mathrm{ZOO}$ & $\mathrm{T}$ & FA-S \\
\hline Moraceae & Brosimum lactescens (S. Moore) C.C. Berg & guarapicica & $\mathrm{ZOO}$ & $\mathrm{T}$ & Amp \\
\hline Moraceae & Ficus adhatodifolia Schott ex Spreng. & & $\mathrm{ZOO}$ & $\mathrm{T}$ & Amp \\
\hline Moraceae & Ficus gomelleira Kunth \& Bouché & figueira-goiaba & $\mathrm{ZOO}$ & $\mathrm{T}$ & Amp \\
\hline Moraceae & Ficus insipida Willd. & figueira & $\mathrm{ZOO}$ & $\mathrm{T}$ & Amp \\
\hline Moraceae & Ficus luschnatiana (Miq.) Miq. & & $\mathrm{ZOO}$ & $\mathrm{T}$ & Amp \\
\hline Moraceae & Ficus organensis (Miq.) Miq. & figueira-miúda & $\mathrm{ZOO}$ & $\mathrm{T}$ & FA-S \\
\hline Moraceae & Ficus sp. & figueira & $\mathrm{ZOO}$ & $\mathrm{T}$ & - \\
\hline Moraceae & Maclura tinctoria (L.) D.Don ex Steud. & tajuva & $\mathrm{ZOO}$ & NT & Amp \\
\hline Moraceae & Pseudolmedia hirtula Kuhlm. & guarapicica & $\mathrm{ZOO}$ & $\mathrm{T}$ & FA-S \\
\hline Moraceae & Sorocea bonplandii (Baill.) W.C.Burger et al. & falsa-espinheira & $\mathrm{ZOO}$ & NT & Amp \\
\hline Myristicaceae & Virola bicuhyba (Schott ex Spreng.) Warb.* & bocuva & $\mathrm{ZOO}$ & NT & FA \\
\hline Myrsinaceae & Ardisia sp. & & $\mathrm{ZOO}$ & $\mathrm{T}$ & - \\
\hline Myrsinaceae & Myrsine coriacea $\mathrm{R}$. Br. & capororoquinha & $\mathrm{ZOO}$ & NT & Amp \\
\hline Myrsinaceae & Myrsine guianensis (Aubl.) Kuntze & & $\mathrm{ZOO}$ & NT & Amp \\
\hline Myrsinaceae & $\begin{array}{l}\text { Myrsine hermogenesii (Jung-Mend. \& Bernacci) } \\
\text { M.F.Freitas \& Kin.-Gouv. }\end{array}$ & capororoca & $\mathrm{ZOO}$ & NT & FA \\
\hline Myrsinaceae & Myrsine umbellata Mart. & capororoca & $\mathrm{ZOO}$ & NT & Amp \\
\hline Myrtaceae & Calyptranthes grandifolia O.Berg & guamirim & $\mathrm{ZOO}$ & $\mathrm{T}$ & FA-S \\
\hline Myrtaceae & Calyptranthes lanceolata O.Berg & guamirim & $\mathrm{ZOO}$ & $\mathrm{T}$ & FA-S \\
\hline
\end{tabular}




\begin{tabular}{|c|c|c|c|c|c|}
\hline Myrtaceae & Calyptranthes lucida Mart. ex DC. & guamirim & $\mathrm{ZOO}$ & $\mathrm{T}$ & Amp \\
\hline Myrtaceae & Calyptranthes strigipes O.Berg & guamirim-cascudo & $\mathrm{ZOO}$ & $\mathrm{T}$ & FA-S \\
\hline Myrtaceae & Campomanesia neriifolia (O.Berg) Nied.* & guavirova & $\mathrm{ZOO}$ & $\mathrm{T}$ & FA-S \\
\hline Myrtaceae & Campomanesia reitziana D.Legrand* & guavirova & $\mathrm{ZOO}$ & $\mathrm{T}$ & FA-S \\
\hline Myrtaceae & Campomanesia xanthocarpa O.Berg & guavirova & $\mathrm{ZOO}$ & NT & FA-S \\
\hline Myrtaceae & Eugenia catharinensis D.Legrand & araçá-angelim & $\mathrm{ZOO}$ & $\mathrm{T}$ & FA-S \\
\hline Myrtaceae & Eugenia cf. brevistyla D.Legrand & & $\mathrm{ZOO}$ & $\mathrm{T}$ & FA-S \\
\hline Myrtaceae & Eugenia $\mathrm{cf}$. burkartiana (D.Legrand) D.Legrand & guapã, guapão & $\mathrm{ZOO}$ & $\mathrm{T}$ & FA-S \\
\hline Myrtaceae & Eugenia cf. candolleana DC. & guamirim-murta & $\mathrm{ZOO}$ & $\mathrm{T}$ & Amp \\
\hline Myrtaceae & Eugenia cf. cerasiflora Miq. & & $\mathrm{ZOO}$ & $\mathrm{T}$ & Amp \\
\hline Myrtaceae & $\begin{array}{l}\text { Eugenia cf. magnibracteolata Mattos \& } \\
\text { D.Legrand }\end{array}$ & & $\mathrm{ZOO}$ & $\mathrm{T}$ & FA-S \\
\hline Myrtaceae & Eugenia $\mathrm{cf}$. uruguayensis Cambess. & & $\mathrm{ZOO}$ & $\mathrm{T}$ & FA-S \\
\hline Myrtaceae & Eugenia malacantha D.Legrand & & $\mathrm{ZOO}$ & $\mathrm{T}$ & FA-S \\
\hline Myrtaceae & Eugenia melanogyna (D.Legrand) Sobral & & $\mathrm{ZOO}$ & $\mathrm{T}$ & FA-S \\
\hline Myrtaceae & Eugenia multicostata D.Legrand & alazão & $\mathrm{ZOO}$ & $\mathrm{T}$ & FA-S \\
\hline Myrtaceae & Eugenia prasina O.Berg* & & $\mathrm{ZOO}$ & $\mathrm{T}$ & FA \\
\hline Myrtaceae & Eugenia sp. 1 & & $\mathrm{ZOO}$ & $\mathrm{T}$ & - \\
\hline Myrtaceae & Eugenia sp. 2 & & $\mathrm{ZOO}$ & $\mathrm{T}$ & - \\
\hline Myrtaceae & Eugenia sp. 3 & guamirim-murta & $\mathrm{ZOO}$ & $\mathrm{T}$ & - \\
\hline Myrtaceae & Eugenia stigmatosa DC. & & $\mathrm{ZOO}$ & NT & FA \\
\hline Myrtaceae & Eugenia subavenia O.Berg & & $\mathrm{ZOO}$ & $\mathrm{T}$ & FA-S \\
\hline Myrtaceae & Eugenia sulcata Spring ex Mart. & & $\mathrm{ZOO}$ & NT & FA-S \\
\hline Myrtaceae & Eugenia umbelliflora O.Berg & guapê-mirim & $\mathrm{ZOO}$ & NT & FA \\
\hline Myrtaceae & $\begin{array}{l}\text { Marlierea eugeniopsoides (D.Legrand \& Kausel) } \\
\text { D.Legrand }\end{array}$ & & $\mathrm{ZOO}$ & $\mathrm{T}$ & FA-S \\
\hline Myrtaceae & Marlierea obscura O.Berg & jaguapiroca & $\mathrm{ZOO}$ & $\mathrm{T}$ & FA-S \\
\hline Myrtaceae & Marlierea reitzii D.Legrand & & $\mathrm{ZOO}$ & $\mathrm{T}$ & FA-S \\
\hline Myrtaceae & Marlierea silvatica (O.Berg) Kiaersk. & guamirim-ferro & $\mathrm{ZOO}$ & $\mathrm{T}$ & FA \\
\hline Myrtaceae & Marlierea tomentosa Cambess. & guapurunga & $\mathrm{ZOO}$ & $\mathrm{T}$ & FA \\
\hline Myrtaceae & $\begin{array}{l}\text { Myrceugenia miersiana (Gardner) D.Legrand \& } \\
\text { Kausel* }\end{array}$ & guamirim & $\mathrm{ZOO}$ & $\mathrm{T}$ & FA \\
\hline Myrtaceae & Myrceugenia myrcioides (Cambess.) O.Berg* & & $\mathrm{ZOO}$ & $\mathrm{T}$ & FA-S \\
\hline Myrtaceae & Myrceugenia reitzii D.Legrand \& Kausel & & $\mathrm{ZOO}$ & $\mathrm{T}$ & FA-S \\
\hline Myrtaceae & Myrcia brasiliensis Kiaersk. & & $\mathrm{ZOO}$ & $\mathrm{T}$ & FA \\
\hline Myrtaceae & Myrcia cf. dichrophylla D.Legrand & & $\mathrm{ZOO}$ & $\mathrm{T}$ & FA-S \\
\hline Myrtaceae & Myrcia flagellaris (D.Legrand) Sobral & guamirim-pitanga & $\mathrm{ZOO}$ & $\mathrm{T}$ & FA-S \\
\hline Myrtaceae & Myrcia glabra (O.Berg) D.Legrand & & $\mathrm{ZOO}$ & $\mathrm{T}$ & FA-S \\
\hline Myrtaceae & Myrcia insularis Gardner & & $\mathrm{ZOO}$ & $\mathrm{T}$ & FA \\
\hline Myrtaceae & Myrcia multiflora (Lam.) DC. & & $\mathrm{ZOO}$ & NT & Amp \\
\hline Myrtaceae & Myrcia palustris DC. & & $\mathrm{ZOO}$ & NT & FA \\
\hline Myrtaceae & Myrcia pubipetala Miq. & & $\mathrm{ZOO}$ & $\mathrm{T}$ & FA-S \\
\hline Myrtaceae & Myrcia pulchra (O.Berg) Kiaersk. & & $\mathrm{ZOO}$ & $\mathrm{T}$ & FA-S \\
\hline Myrtaceae & Myrcia racemosa (O.Berg) Kiaersk. & & $\mathrm{ZOO}$ & NT & Amp \\
\hline Myrtaceae & Myrcia richardiana Kiaersk. & & $\mathrm{ZOO}$ & $\mathrm{T}$ & FA-S \\
\hline Myrtaceae & Myrcia spectabilis DC. & guamirim-ameixa & $\mathrm{ZOO}$ & $\mathrm{T}$ & FA \\
\hline Myrtaceae & Myrcia splendens (Sw.) DC. & & $\mathrm{ZOO}$ & NT & Amp \\
\hline Myrtaceae & Myrcia tenuivenosa Kiaersk. & & $\mathrm{ZOO}$ & $\mathrm{T}$ & FA-S \\
\hline Myrtaceae & Myrcia tijucensis Kiaersk. & & $\mathrm{ZOO}$ & $\mathrm{T}$ & FA-S \\
\hline Myrtaceae & Myrciaria floribunda (Willd.) O.Berg & & $\mathrm{ZOO}$ & NT & Amp \\
\hline Myrtaceae & Myrtaceae 1 & & $\mathrm{ZOO}$ & NT & - \\
\hline Myrtaceae & Myrtaceae 2 & & $\mathrm{ZOO}$ & $\mathrm{T}$ & - \\
\hline Myrtaceae & Myrtaceae 3 & & $\mathrm{ZOO}$ & $\mathrm{T}$ & - \\
\hline Myrtaceae & Myrtaceae 4 & & $\mathrm{ZOO}$ & NT & - \\
\hline Myrtaceae & Myrtaceae 5 & & $\mathrm{ZOO}$ & $\mathrm{T}$ & - \\
\hline
\end{tabular}




\begin{tabular}{|c|c|c|c|c|c|}
\hline Myrtaceae & Myrtaceae 6 & & $\mathrm{ZOO}$ & $\mathrm{T}$ & - \\
\hline Myrtaceae & Myrtaceae 7 & & $\mathrm{ZOO}$ & $\mathrm{T}$ & - \\
\hline Myrtaceae & $\begin{array}{l}\text { Neomitranthes glomerata (D.Legrand) } \\
\text { D.Legrand }\end{array}$ & guamirim-ferro & $\mathrm{ZOO}$ & NT & FA-S \\
\hline Myrtaceae & Plinia edulis (Vell.) Sobral & cambucá & $\mathrm{ZOO}$ & $\mathrm{T}$ & FA-S \\
\hline Myrtaceae & Plinia sp. & jabuticabeira & $\mathrm{ZOO}$ & $\mathrm{T}$ & - \\
\hline Myrtaceae & Psidium cattleianum Sabine & araçá & $\mathrm{ZOO}$ & NT & Amp \\
\hline Nyctaginaceae & Guapira hirsuta (Choisy) Lundell & & $\mathrm{ZOO}$ & $\mathrm{T}$ & Amp \\
\hline Nyctaginaceae & Guapira opposita (Vell.) Reitz & sebeiro & $\mathrm{ZOO}$ & NT & Amp \\
\hline Nyctaginaceae & Pisonia ambigua Heimerl & laranjeira & AUT & NT & Amp \\
\hline Ochnaceae & Ouratea parviflora (DC.) Baill. & parapari & $\mathrm{ZOO}$ & $\mathrm{T}$ & FA \\
\hline Olacaceae & Heisteria silvianii Schwacke & & $\mathrm{ZOO}$ & $\mathrm{T}$ & Amp \\
\hline Olacaceae & Tetrastylidium grandifolium (Baill.) Sleum. & & $\mathrm{ZOO}$ & $\mathrm{T}$ & FA \\
\hline Penthaphyllaceae & Ternstroemia brasiliensis Cambess. & & $\mathrm{ZOO}$ & NT & Amp \\
\hline Peraceae & Pera glabrata (Schott) Baill. & tabocuva & $\mathrm{ZOO}$ & NT & Amp \\
\hline Phyllantaceae & Hyeronima alchorneoides Allemão & licurana & $\mathrm{ZOO}$ & NT & Amp \\
\hline Phyllantaceae & Margaritaria nobilis L.f. & & $\mathrm{ZOO}$ & NT & Amp \\
\hline Phytolaccaceae & Phytolacca dioica $\mathrm{L}$. & ceboleiro & $\mathrm{ZOO}$ & NT & Amp \\
\hline Phytolaccaceae & Seguieria langsdorfii Moq. & laranjeira-de-espinho & ANE & NT & Amp \\
\hline Picramniaceae & Picramnia ramiflora Planch. & & $\mathrm{ZOO}$ & NT & Amp \\
\hline Piperaceae & Piper aduncum L. & & $\mathrm{ZOO}$ & NT & Amp \\
\hline Piperaceae & Piper arboreum Aubl. & & $\mathrm{ZOO}$ & $\mathrm{T}$ & Amp \\
\hline Piperaceae & Piper cernuum Vell. & & $\mathrm{ZOO}$ & $\mathrm{T}$ & Amp \\
\hline Piperaceae & Piper gaudichaudianum Kunth & & $\mathrm{ZOO}$ & NT & Amp \\
\hline Piperaceae & Piper malacophyllum C. DC. & & $\mathrm{ZOO}$ & $\mathrm{T}$ & Amp \\
\hline Podocarpaceae & Podocarpus sellowii Klotzsch ex Endl. & pinheirinho & $\mathrm{ZOO}$ & $\mathrm{T}$ & - \\
\hline Polygonaceae & Coccoloba sp. & & $\mathrm{ZOO}$ & NT & - \\
\hline Polygonaceae & Coccoloba warmingii Meisn. & & $\mathrm{ZOO}$ & NT & Amp \\
\hline Proteaceae & Roupala montana Aubl. & carvalho & ANE & $\mathrm{T}$ & Amp \\
\hline Quiinaceae & Quiina glaziovii Engl. & quina, jorovarana & $\mathrm{ZOO}$ & $\mathrm{T}$ & FA \\
\hline Rosaceae & Prunus myrtifolia (L.) Urb. & pessegueiro-bravo & $\mathrm{ZOO}$ & NT & Amp \\
\hline Rubiaceae & Alseis floribunda Schott & tarumazinho & ANE & $\mathrm{T}$ & Amp \\
\hline Rubiaceae & Amaioua guianensis Aubl. & pau-carvão & $\mathrm{ZOO}$ & NT & Amp \\
\hline Rubiaceae & Bathysa australis (A.St.-Hil.) K.Schum. & cajujão & AUT & $\mathrm{T}$ & FA \\
\hline Rubiaceae & Cordiera concolor (Cham.) Kuntze & & $\mathrm{ZOO}$ & $\mathrm{T}$ & Amp \\
\hline Rubiaceae & Coussarea contracta Benth. \& Hook f. & & $\mathrm{ZOO}$ & NT & Amp \\
\hline Rubiaceae & Posoqueria latifolia Roem. \& Schult. & laranjeira-de-macaco & $\mathrm{ZOO}$ & NT & Amp \\
\hline Rubiaceae & Psychotria nuda (Cham. \& Schltdl.) Wawra & pasto-de-anta & $\mathrm{ZOO}$ & $\mathrm{T}$ & FA-S \\
\hline Rubiaceae & Psychotria sp. 1 & pasto-de-anta & $\mathrm{ZOO}$ & $\mathrm{T}$ & - \\
\hline Rubiaceae & Psychotria sp. 2 & pasto-de-anta & $\mathrm{ZOO}$ & $\mathrm{T}$ & - \\
\hline Rubiaceae & Randia armata (Sw.) DC. & & $\mathrm{ZOO}$ & NT & Amp \\
\hline Rubiaceae & Rudgea recurva Müll. Arg. & & $\mathrm{ZOO}$ & $\mathrm{T}$ & FA-S \\
\hline Rutaceae & Esenbeckia grandiflora Mart. & & AUT & $\mathrm{T}$ & Amp \\
\hline Rutaceae & Metrodorea nigra A. St.-Hil. & & AUT & $\mathrm{T}$ & Amp \\
\hline Rutaceae & Pilocarpus pauciflorus A. St.-Hil. & coração-de-negro & AUT & NT & FA \\
\hline Rutaceae & Zanthoxylum rhoifolium Lam. & mamica-de-porca & $\mathrm{ZOO}$ & NT & Amp \\
\hline Sabiaceae & Meliosma sellowii Urb. & & $\mathrm{ZOO}$ & $\mathrm{T}$ & Amp \\
\hline Salicaceae & Casearia decandra Jacq. & guassatunga & $\mathrm{ZOO}$ & $\mathrm{T}$ & Amp \\
\hline Salicaceae & Casearia obliqua Spreng. & guassatunga & $\mathrm{ZOO}$ & $\mathrm{T}$ & Amp \\
\hline Salicaceae & Casearia sylvestris $\mathrm{Sw}$. & guassatunga & $\mathrm{ZOO}$ & NT & Amp \\
\hline Sapindaceae & $\begin{array}{l}\text { Allophylus edulis (A. St.-Hil. et al.) Hieron. ex } \\
\text { Niederl. }\end{array}$ & vacum & $\mathrm{ZOO}$ & $\mathrm{T}$ & Amp \\
\hline Sapindaceae & Allophylus petiolatus Radlk. ex Wihl.Muller. & vacum & $\mathrm{ZOO}$ & $\mathrm{T}$ & FA \\
\hline Sapindaceae & Allophylus puberulus Radlk. & vacum & $\mathrm{ZOO}$ & $\mathrm{T}$ & FA \\
\hline Sapindaceae & Cupania oblongifolia Mart. & cuvatã & $\mathrm{ZOO}$ & NT & Amp \\
\hline
\end{tabular}




\begin{tabular}{|c|c|c|c|c|c|}
\hline Sapindaceae & Matayba guianensis Aubl. & miguel-pintado & $\mathrm{ZOO}$ & NT & Amp \\
\hline Sapindaceae & Matayba juglandifolia Radlk. & cuvatã & $\mathrm{ZOO}$ & NT & Amp \\
\hline Sapotaceae & Chrysophyllum inornatum Mart.* & sambaqui, murta & $\mathrm{ZOO}$ & NT & FA \\
\hline Sapotaceae & Chrysophyllum sp. & & $\mathrm{ZOO}$ & $\mathrm{T}$ & - \\
\hline Sapotaceae & Chrysophyllum viride Mart. \& Eichler* & & $\mathrm{ZOO}$ & $\mathrm{T}$ & FA \\
\hline Sapotaceae & Manilkara subsericea Dubard* & maçaranduba & $\mathrm{ZOO}$ & $\mathrm{T}$ & FA-S \\
\hline Sapotaceae & Pouteria sp. & guapeva & $\mathrm{ZOO}$ & $\mathrm{T}$ & - \\
\hline Sapotaceae & Pouteria torta (Mart.) Radlk. & guapeva & $\mathrm{ZOO}$ & $\mathrm{T}$ & Amp \\
\hline Sapotaceae & Pouteria venosa (Mart.) Baehni & & $\mathrm{ZOO}$ & $\mathrm{T}$ & Amp \\
\hline Sapotaceae & Pradosia lactescens Radlk. & & $\mathrm{ZOO}$ & $\mathrm{T}$ & FA \\
\hline Solanaceae & Acnistus arborescens Schltdl. & barrileira & $\mathrm{ZOO}$ & NT & FA \\
\hline Solanaceae & Cestrum intermedium Sendtn. & & $\mathrm{ZOO}$ & NT & FA \\
\hline Solanaceae & Solanum pseudoquina A. St.-Hil.* & quina-brava & $\mathrm{ZOO}$ & NT & FA \\
\hline Symplocaceae & Symplocos estrellensis Casar. & & $\mathrm{ZOO}$ & NT & FA \\
\hline Symplocaceae & Symplocos laxiflora Benth. & vanvu & $\mathrm{ZOO}$ & $\mathrm{T}$ & FA-S \\
\hline Theaceae & Laplacea fruticosa (Schrad.) Kobuski & & $\mathrm{ZOO}$ & $\mathrm{T}$ & Amp \\
\hline Thymelaeaceae & Daphnopsis cf. fasciculata (Meisn.) Nevling & embira & $\mathrm{ZOO}$ & $\mathrm{T}$ & Amp \\
\hline Urticaceae & Bohemeria caudata $\mathrm{Sw}$. & & AUT & NT & Amp \\
\hline Urticaceae & Cecropia glaziovi Snethl. & embaúba & $\mathrm{ZOO}$ & NT & FA \\
\hline Urticaceae & Cecropia pachystachya Trécul & embaúba & $\mathrm{ZOO}$ & NT & Amp \\
\hline Urticaceae & Coussapoa microcarpa (Schott) Rizzini & mata-pau & $\mathrm{ZOO}$ & $\mathrm{T}$ & Amp \\
\hline Urticaceae & Pourouma guianensis Aubl. & embaubarana & $\mathrm{ZOO}$ & NT & Amp \\
\hline Verbenaceae & Cytharexylum myrianthum Cham. & jacataúva & $\mathrm{ZOO}$ & NT & Amp \\
\hline Vochysiaceae & Vochysia bifalcata Warm. & guaricica & ANE & NT & FA-S \\
\hline
\end{tabular}

Considerando a riqueza arbórea encontrada em levantamentos de diferentes trechos de Floresta Atlântica da costa sul/sudeste brasileira, percebe-se que há um padrão comum de distribuição das famílias com maior riqueza específica. Das famílias mais ricas evidenciadas nesse levantamento (Myrtaceae, Fabaceae, Lauraceae, Melastomatacea, Rubiaceae e Moraceae), raramente uma ou outra delas não figura entre as mais ricas em outros levantamentos realizados em Floresta Ombrófila Densa, desde aquelas localizadas em trechos mais ao norte, como no Espírito Santo e Rio de Janeiro, até os levantamentos efetuados em solos catarinenses (ARAGAKI; MANTOVANI, 1998; SANCHEZ et al., 1999; DIAS et al., 1995, 2000; GOMES et al., 2000; OLIVEIRA, 2002; ASSIS et al., 2004; MANTOVANI et al., 2005; NEGRELLE, 2006; BLUM, 2006; CARVALHO et al., 2007; CERVI et al., 2007; SCHORN; GALVÃO, 2009). Esse padrão também foi constatado por Tabarelli e Mantovani (1999) e por Murray-Smith et al. (2009) em compilações de levantamentos realizados na costa brasileira.

Myrtaceae englobou 19,7\% de todas as espécies encontradas na RNRC. Essa riqueza elevada corrobora as afirmações feitas por vários estudiosos, que destacam a importância da família tanto no conjunto florístico da Floresta Atlântica (MORI et al., 1983; TABARELLI; MANTOVANI, 1999; OLIVEIRA-FILHO; FONTES, 2000; CATHARINO et al., 2006; BLUM, 2006), como em formações neotropicais (CHAZDON; DENSLOW, 2002), respaldando seu caráter endêmico (SYTSMA et al., 2004), muito em função de a costa brasileira ser um dos centros de riqueza da família (TABARELLI; MANTOVANI, 1999; CEPVMA, 2010).

A zoocoria é a síndrome mais comum, sendo exclusiva para Myrtaceae, Lauraceae, Annonaceae, Moraceae, Sapindaceae, Arecaceae e Sapotaceae, e está presente em mais de $80 \%$ das espécies de Rubiaceae e Melastomataceae, todas elas de alta riqueza na Floresta Atlântica. Essa maior representatividade da zoocoria é comum em levantamentos realizados em florestas da costa brasileira, destacando-se especialmente na sinúsia arbórea, como constatado por Zipparro et al. (2005) e Marques e Oliveira (2005). Fabaceae, ao contrário das anteriores, possui a anemocoria como síndrome mais importante, constatada em $40,6 \%$ dos taxa registrados, seguida pela zoocoria $(31,3 \%)$ e pela autocoria $(28,1 \%)$. Essa família é especialmente importante para a primeira e a última síndromes, já que esses valores representam $37,1 \%$ das espécies anemocóricas e 40,9\% das autocóricas desse estudo.

Com respeito à distribuição geográfica, quase metade das espécies $(48,4 \%)$ foi enquadrada como de ampla distribuição, 18,3\% podem ser encontradas ao longo de todo o trecho de ocorrência da Floresta 
Atlântica no Brasil e 23,2\% estão restritas à porção sul/sudeste dessa formação. Taxa identificados em nível genérico não foram classificados e representaram $10,1 \%$ do total encontrado. A concentração de espécies de ampla distribuição também foi retratada por Reginato e Goldenberg (2007), que estudaram um trecho transicional entre Floresta Atlântica e Floresta com Araucária. Destaca-se aqui a representatividade de espécies de distribuição restrita ao sul/sudeste da Floresta Atlântica, representadas, entre outras, por Attalea dubia, Buchenavia kleinii, Cryptocarya aschersoniana, Eugenia multicostata, Ficus organensis, Manilkara subsericea, Miconia cabucu, Myrcia pubipetala, Ocotea catharinensis, Protium kleinii e Vochysia bifalcata, que reforçam a congruência com a ideia de que o bioma ao qual a floresta pertence possui pelo menos duas regiões biogeográficas bem delimitadas, uma ao sul e outra ao norte do vale do rio Doce, no Espírito Santo (SILVA et al., 2004).

Historicamente, os trechos de planície da Floresta Atlântica foram os mais utilizados pelo homem (BUDOWSKI, 1966), tendo em vista as condições mais adequadas para a implantação de sistemas produtivos encontradas nos solos derivados de sedimentos aluviais. Essa ocupação resultou na degradação da floresta de forma mais intensa (BRITEZ et al., 2006), situação comprovada pela menor extensão de áreas bem conservadas nesses trechos da Floresta Atlântica. Esse cenário explica parcialmente o número significativo de espécies registradas na área que constam em listas de plantas ameaçadas de extinção $(6,6 \%)$, comparada a menos de $2 \%$ estimados para a formação no Brasil como um todo (TABARELLI et al., 2003). A isso, soma-se o atual estado de conservação da cobertura da Floresta Atlântica, que assistiu a diversos ciclos econômicos e passou por processos de degradação variados, desde a extração seletiva de madeira até os sistemas de produção extensiva (especialmente bubalinocultura e orizicultura) e a produção de banana (DEAN, 1996), que contribuem ainda mais para a alteração ambiental. Ainda que hoje alguns trechos do bioma estejam oficialmente protegidos e as taxas de desmatamento, se comparadas a apenas algumas décadas atrás (MAACK, 1968; FUNDAÇÃO SOS MATA ATLÂNTICA; INPE, 2008), tenham diminuído significativamente, os remanescentes florestais que restaram estão extremamente fragmentados e continuam sendo alvo de perturbações que podem interferir na manutenção de populações vegetais e animais. Associado a isso, a superexplotação de várias essências nativas, muitas delas espécies-chave (GALLETTI et al., 1999), de fundamental importância para a manutenção da organização e da diversidade das comunidades de fauna e de flora (MILLS et al., 1993; PAINE, 1995), como o palmito-juçara (Euterpe edulis) ou as canelas (Ocotea catharinensis, O. odorifera e $O$. puberula), causa efeitos de depleção em cascata, levando a uma condição de fragilidade ambiental que demanda ações efetivas de conservação do bioma.

\section{CONCLUSÕES}

- Com base no levantamento florístico do componente arbóreo de um trecho de Floresta Ombrófila Densa da Reserva Natural do Rio Cachoeira, é possível concluir que, apesar dos antropismos, a vegetação que persiste reserva é extremamente variada, dispondo de uma riqueza típica de formações tropicais. Há distinção na forma de dispersão, sendo que grande parte das espécies utiliza-se de vetores biológicos nesse processo. $\mathrm{O}$ mesmo não acontece para a categorização ecológica, em que o número de espécies tolerantes e não tolerantes à sombra é praticamente o mesmo. Em termos de distribuição geográfica, espécies restritas à Floresta Attântica representam quase metade do universo florístico, sendo que, dentro desse limite fitofisionômico, aquelas com distribuição mais ao sul são pouco mais numerosas $(56 \%)$.

- A ocorrência de uma quantidade expressiva de espécies sob algum grau de ameaça de extinção corrobora a condição de bioma ameaçado e justifica ações imediatas e efetivas de conservação nessa que é uma das últimas áreas contínuas de Floresta Atlântica no Brasil.

\section{AGRADECIMENTOS}

Ao Department of Energy USA, pelo financiamento DE-FC26-01NT411151; aos funcionários da Reserva Cachoeira e aos colegas da equipe de Monitoramento de Carbono, pelo suporte ao longo do trabalho; aos especialistas Gert Hatschbach, Osmar Ribas, Marcos Sobral e Renato Goldenberg, pelas identificações; a Armando C. Cervi, Luciano F. Watzlawick, Márcia C. M. Marques e Ricardo M. Britez, pelas sugestões ao manuscrito. 


\section{REFERÊNCIAS}

ANGIOSPERM PHYLOGENY GROUP (APG). An update of the Angiosperm Phylogeny Group classification for the orders and families of flowering plants. Botanical Journal of the Linnean Society, n. 161 , p. $105-121,2009$.

ARAGAKI, S.; MANTOVANI, W. Caracterização do clima e da vegetação de remanescente florestal no Planalto Paulistano (SP). In: SIMPÓSIO DE ECOSSISTEMAS DA COSTA BRASILEIRA, 4., 1998, São Paulo. Anais do... São Paulo: Academia de Ciências do Estado de São Paulo, 1998. p. 25 - 36.

ASSIS, A. M.; PEREIRA, O. J.; THOMAZ, L. D. Fitossociologia de uma floresta de restinga no Parque Estadual Paulo César Vinha, Setiba, município de Guarapari (ES). Revista Brasileira de Botânica, v. 27, n. 2, p. 349 - 361, 2004.

BLUM, C. T. A Floresta Ombrófila Densa na Serra da Prata, Parque Nacional Saint-Hilaire/Lange, PR - caracterização florística, fitossociológica e ambiental de um gradiente altitudinal. $195 \mathrm{f}$. Dissertação (Mestrado) - Universidade Federal do Paraná, Curitiba, 2006.

BRASIL. Ministério do Meio Ambiente (MMA). Instrução Normativa n. 6, de 23 de setembro de 2008. Diário Oficial da União, 24 set. 2008. Disponível em: <http://portal.saude.gov.br/portal/arquivos/pdf/MMA_IN_N_6.pdf>. Acesso em: 25/10/2011.

BRITEZ, R. M.; BORGO, M.; TIEPOLO, G.; FERRETTI, A. R.; CALMON, M.; HIGA, R. Estoque e incremento de carbono em florestas e povoamentos de espécies arbóreas com ênfase na Floresta Atlântica do sul do Brasil. Colombo: EMBRAPA, 2006. 165 p.

BUDOWSKI, G. Los bosques de los trópicos húmedos de América. Turrialba, v. 16, n. 3, p. 278 - 285, 1966.

CARVALHO, F. A.; NASCIMENTO, M. T.; BRAGA, J. M. A. Estrutura e composição florística do estrato arbóreo de um remanescente de Mata Atlântica Submontana no município de Rio Bonito, RJ, Brasil (Mata Rio Vermelho). Revista Árvore, v. 31, n. 4, p. 717 - 730, 2007.

CATHARINO, E. L.; BERNACCI, L. C.; FRANCO, G. A. D. C.; DURIGAN, G.; METZGER, J. P. Aspectos da composição e diversidade do componente arbóreo das florestas da Reserva Florestal do Morro Grande, Cotia, SP. Biota Neotropica, v. 6, 2006. Disponível em: <http://www.biotaneotropica .org.br/v6n2/pt/abstract?article+bn00306022006>. Acesso em: 10/02/2010.

CENTROS DE ENDEMISMO DE PLANTAS VASCULARES NA MATA ATLÂNTICA (CEPVMA). Endemismos - angiospermas. Disponível em: 〈http://sagui.icb.ufmg.br/bot/mataatlantica/endangio.htm> Acesso em: 10/01/2010.

CERVI, A. C.; HATSCHBACH, G. G.; LINSINGEN, L. Composição florística de um trecho de Floresta Ombrófila Densa de Terras Baixas (Floresta Atlântica) na Reserva Ecológica de Sapitanduva (Morretes, Paraná, Brasil). Fontqueria, v. 55, n. 52, p. 423 - 438, 2007.

CHAZDON, R. L.; DENSLOW, J. S. Floristic composition and species richness. In: CHAZDON, R. L.; WHITMORE, T. C. (Ed.). Foundations of Tropical Rainforest Biology: classic papers with commentaries. Chicago: University of Chicago Press, 2002. p. 513 - 522.

DEAN, W. A ferro e fogo: a história e a devastação da Mata Atlântica brasileira. São Paulo: Companhia das Letras, 1996. 484 p.

DIAS, A. C.; CUSTÓDIO FILHO, A.; FRANCO, G. A. D. C. Diversidade do componente arbóreo em um trecho de floresta secundária, Parque Estadual de Carlos Botelho, SP. Revista do Instituto Florestal, v. 12 , n. 2, p. $127-153,2000$.

Diversidade do componente arbóreo em floresta pluvial atlântica secundária, São Paulo, Brasil. Revista do Instituto Florestal, v. 12, p. 125 - 155, 1995. 
DITTRICH, V. A.; WAECHTER, J. L.; SALINO, A. Species richness of pteridophytes in a montane Atlantic rain forest plot of Southern Brazil. Acta Botanica Brasilica, v. 19, n. 3, p. 519 - 525, 2005.

FIASCHI, P.; PIRANI, J. R. Review of plant biogeographic studies in Brazil. Journal of Systematics and Evolution, v. 0, n. 0, p. 1 - 20, 2009.

FUNDAÇÃO SOS MATA ATLÂNTICA; INSTITUTO NACIONAL DE PESQUISAS ESPACIAIS (INPE). Atlas dos remanescentes florestais da Mata Atlântica período 2008-2010. São Paulo: SOS Mata Atlântica; INPE, 2008. 60 p.

GALlETTI, M.; ZIPARRO, V. B.; MORELLATO, L. P. C. Fruiting phenology and frugivory on the palm Euterpe edulis in a lowland Atlantic Forest of Brazil. Ecotropica, v. 5, p. 115 - 122, 1999.

GIULIETTI, A. M.; HARLEY, R. M.; QUEIROZ, L. P.; WANDERLEY, M. G. L.; VAN DEN BERG, C. Biodiversidade e conservação das plantas no Brasil. Megadiversidade, v. 1, n. 1, p. 52 - 61, 2005.

GOMES, E. P. C.; MANTOVANI, W.; KAWALL, M. Estrutura e status sucessional de florestas na bacia do rio Ribeira de Iguape, Vale do Ribeira, SP. In: SIMPÓSIO DE ECOSSISTEMAS BRASILEIROS, 2., 2000. Anais do... IESP, 2000. v. 109, p. 170 - 183.

GUAPYASSÚ, M. S. Caracterização fitossociológica de três fases sucessionais de uma Floresta Ombrófila Densa Submontana, Morretes - Paraná. 165 f. Dissertação (Mestrado) - Universidade Federal do Paraná, Curitiba, 1994.

HARTSHORN, G. S. Treefalls and tropical forest dynamics. In: TOMLINSON, P. B.; ZIMMERMANN, Z. Z. (Ed.). Tropical trees as living systems. New York: Cambridge University Press, 1978. p. 617 638.

INSTITUTO BRASILEIRO DE GEOGRAFIA E ESTATÍSTICA (IBGE). Manual técnico da vegetação brasileira. Rio de Janeiro: Fundação Inst. Brasileiro de Geografia e Estatística/DERNA, 1992. 92 p. (Manuais Técnicos de Geociências 1).

INSTITUTO PARANAENSE DE DESENVOLVIMENTO ECONÔMICO E SOCIAL (IPARDES). Zoneamento da Área de Proteção Ambiental de Guaraqueçaba. Curitiba: IPARDES, 2001. 150 p.

INTERNATIONAL UNION FOR CONSERVATION OF NATURE (IUCN). IUCN red list of threatened species. Version 2011.1. Disponível em: <www.iucnredlist.org>. Acesso em: 13/07/2011.

JARDIM BOTÂNICO DO RIO DE JANEIRO (JBRJ). Lista de espécies da flora do Brasil. Disponível em: <http://floradobrasil.jbrj.gov.br/2010/>. Acesso em: 25/11/2010.

KOZERA, C.; RODRIGUES, R. R.; DITTRICH, V. A. O. Composição florística do sub-bosque de uma Floresta Ombrófila Densa Montana, Morretes, PR, Brasil. Floresta, v. 39, n. 2, p. 323 - 334, 2009.

LEITE, P.; KLEIN, R. M. Vegetação. In: IBGE. Geografia do Brasil: região Sul. Rio de Janeiro: Instituto Brasileiro de Geografia e Estatística, 1990. v. 2. p. 113 - 150.

MAACK, R. Geografia física do Estado do Paraná. Curitiba: Banco de Desenvolvimento do Paraná; Universidade Federal do Paraná; Instituto de Biologia e Pesquisa Tecnológica, 1968. 350 p.

MANTOVANI, M.; RUSCHEL, A. R.; PUCHALSKI, A.; SILVA, J. Z.; REIS, M. S.; NODARI, R. Diversidade de espécies e estrutura sucessional de uma formação secundária da Floresta Ombrófila Densa. Scientia Forestalis, v. 67, p. 14 - 26, 2005.

MARQUES, M. C. M.; OLIVEIRA, P. E. A. M. Características reprodutivas das espécies vegetais da planície litorânea. In: MARQUES, M. C. M.; BRITEZ, R. M. (Org.). História natural e conservação da Ilha do Mel. Curitiba: Editora da Universidade Federal do Paraná, 2005. 266 p.

MILLS, S.; SOULE, M. E.; DOAK, D. F. The keystone-species concept in ecology and conservation. BioScience, v. 43, n. 4, p. 219 - 227, 1993. 
MORI, S. A.; BOOM, B. M.; CARVALHO, A. M.; SANTOS, T. S. Ecological importance of Myrtaceae in an Eastern Brazilian wet forest. Biotropica, v. 15, p. 68 - 70, 1983.

MUELLER-DOMBOIS, D.; ELLENBERG, H. Aims and methods of vegetation ecology. New York: John Wiley \& Sons, 1974. 547 p.

MURRAY-SMITH, C.; BRUMMITT, N. A.; OLIVEIRA-FILHO, A. T.; BACHMAN, S.; MOAT, J.; LUGHADHA, E. M. N.; LUCAS, E. J. Plant diversity hotspots in the Atlantic Coastal Forests of Brazil. Conservation Biology, v. 23, n. 1, p. 151 - 163, 2009.

MYERS, N.; MITTERMEIER, R. A.; MITTERMEIER, C. G.; FONSECA, G. A. B.; KENT, J. Biodiversity hotspots for conservation priorities. Nature, v. 403, p. 853 - 858, 2000.

NEGRELLE, R. R. B. Composição florística e estrutura vertical de um trecho de Floresta Ombrófila Densa de Planície Quaternária. Hoehnea, v. 33, n. 3, p. 261 - 289, 2006.

OLIVEIRA, R. R. Ação antrópica e resultantes sobre a estrutura e composição da Mata Atlântica na Ilha Grande, RJ. Rodriguésia, v. 53, n. 82, p. 33 - 58, 2002.

OLIVEIRA-FILHO, A. T.; FONTES, M. A. L. Patterns of floristic differentiation among Atlantic Forests in Southeastern Brazil and the influence of climate. Biotropica, v. 32, p. $793-810,2000$.

PAINE, R. T. A conversation on refining the concept of keystone species. Conservation Biology, v. 9, n. 4, p. 962 - 964, 1995.

PINTO, L. P.; BRITO, M. C. W. Dynamics of biodiversity loss in the Brazilian Atlantic Forest: an introduction. In: GALINDO-LEAL; CÂMARA, I. G (Ed.). The Atlantic Forest of South America biodiversity status, threats and outlook. Washington: Island Press, 2003. p. 27 - 30.

PRÓ-ATLÂNTICA. Programa Proteção da Mata Atlântica. Atlas da Floresta Atlântica no Paraná área de abrangência do programa proteção da Floresta Atlântica. Curitiba: SEMA/Programa Proteção da Floresta Atlântica, 2005. 104 p.

REGINATO, M.; GOLDENBERG, R. Análise florística, estrutural e fitogeográfica da vegetação em região de transição entre as Florestas Ombrófilas Mista e Densa Montana, Piraquara, Paraná, Brasil. Hoehnea, v. 34, n. 3, p. 349 - 364, 2007.

RIBEIRO, M. C.; METZGER, J. P.; MARTENSEN, A. C.; PONZONI, F. J.; HIROTA, M. M. The Brazilian Atlantic Forest: how much is left, and how is the remaining forest distributed? Implications for conservation. Biological Conservation, v. 142, p. 1141 - 1153, 2009.

RODERJAN, C. V. O gradiente da Floresta Ombrófila Densa no Morro do Anhangava, Quatro Barras, PR - aspectos climáticos, pedológicos e fitossociológicos. 119 p. Tese (Doutorado) Universidade Federal do Paraná, Curitiba, 1994.

SANCHEZ, M.; PEDRONI, F.; LEITÃO-FILHO, H. F. Composição florística de um trecho de floresta ripária na Mata Atlântica em Picinguaba, Ubatuba, SP. Revista Brasileira de Botânica, v. 22, n. 1, p. 31 - 42, 1999.

SCHORN, L. A. Levantamento florístico e análise estrutural em três unidades edáficas em uma Floresta Ombrófila Densa Montana no estado do Paraná. 144 f. Dissertação (Mestrado) Universidade Federal do Paraná, Curitiba, 1992.

SCHORN, L. A.; GALVÃO, F. Dinâmica do estrato arbóreo em três estádios sucessionais de uma Floresta Ombrófila Densa em Blumenau, SC. Cerne, v. 5, n. 2, p. 221 - 235, 2009.

SILVA, F. C. Composição florística e estrutura fitossociológica da floresta tropical ombrófila da encosta atlântica no município de Morretes, estado do Paraná. Acta Biologica Paranaense, v. 23, n. 1, 2, 3, 4, p. 1 - 54, 1994.

SILVA, J. M. C.; SOUSA, M. C.; CASTELlETTI, C. H. M. Areas of endemism for passerine birds in Atlantic Forest, South America. Global Ecology and Biogeography, v. 13, p. 85 - 92, 2004. 
SOCIEDADE DE PESQUISA EM VIDA SELVAGEM E EDUCAÇÃO AMBIENTAL (SPVS); THE NATURE CONSERVANCY (TNC). Levantamento de solos - Reserva Natural do Rio Cachoeira, 2002. 193 p. Relatório técnico.

STELLFELD, C. Fitogeografia geral do estado do Paraná. Arquivos do Museu Paranaense, v. 7, p. 309 - 361, 1949.

SYTSMA, K. J.; LITT, A.; ZJHRA. M. L.; PIRES. J. C.; NEPOKROEFF, M.; CONTI, E.; WALKER, J.; WILSON. P. G. Clades, clocks, and continents: historical and biogeographical analysis of Myrtaceae, Vochysiaceae, and relatives in the southern hemisphere. International Journal of Plant Sciences, v. 165 , p. 85 - 105, 2004. (4 Suppl.).

TABARELli, M.; PINTO, L. P.; SILVA, J. M. C.; COSTA, C. M. R. Endangered species and conservation planning. In: GALINDO-LEAL; CÂMARA, I. G. (Ed.). The Atlantic Forest of South America - biodiversity status, threats and outlook. Washington: Island Press, 2003. p. 86 - 94.

TABARELLI, M.; MANTOVANI, W. A riqueza de espécies arbóreas na floresta atlântica de encosta no estado de São Paulo (Brasil). Revista Brasileira de Botânica, v. 22, n. 2, p. 217 - 223, 1999.

VAN DER PIJL, L. Principles of dispersal in higher plants. 3. ed. Berlin: Springer-Verlag, 1982.

ZIPPARRO, V. B.; GUILHERME, F. A. G.; ALMEIDA-SCABBIA, R. J.; MORELLATO, L. P. C. Levantamento florístico de floresta atlântica no sul do estado de São Paulo, Parque Estadual Intervales, Base Saibadela. Biota Neotropica, v. 5, n. 1., 2005. Disponível em: <http://www.biotaneotropica.org.br/ v5n1/pt/abstract?inventory+BN026>. Acesso em: 10/02/2010. 